\title{
Consensus and evidence-based medication review to optimize and potentially reduce psychotropic drug prescription in institutionalized dementia patients
}

Mireia Massot Mesquida ${ }^{1 *}$ D, Montserrat Tristany Casas $^{2}$, Alicia Franzi Sisó ${ }^{1}$, Isabel García Muñoz ${ }^{1}$ Óscar Hernández Vian ${ }^{1}$ and Pere Torán Monserrat ${ }^{3}$ (D)

\begin{abstract}
Background: Dementia patients often show neuropsychiatric symptoms, known as behavioral and psychological symptoms of dementia (BPSD). These are a common motive for medical consultations, hospitalizations, and nursing home stays. Various studies have suggested that the high prevalence of psychotropic drug use to treat BPSD in institutionalized dementia patients may lead to impaired cognitive capacity, rigidity, somnolence, and other complications during the course of the illness. The aim of this study was to design a consensus-based intervention between care levels to optimize and potentially reduce prescription of psychotropic drugs in institutionalized patients with dementia and assess the changes occurring following its implementation.

Methods: Design: Prospective, quasi-experimental, pre/post intervention, multicenter study. Scope: 7 nursing homes associated with a single primary care team. Inclusion Criteria: Institutionalized patients diagnosed with dementia and under treatment with 1 or more psychotropic drugs for at least 3 months. Sample: 240 individuals; mean age, 87 years (SD: 6.795); 75\% (180) women. Intervention: Creation of evidence-based therapeutic guidelines for psychotropic drug use in the treatment of BPSD by consensus between reference professionals. Joint review (primary care and geriatric care nursing home professionals) of the medication based on the guidelines and focusing on individual patient needs. Primary variable: Number of psychotropic drugs used per patient. Assessment: Preintervention, immediate postintervention, and at 1 and 6 months.

Results: Overall, the number of psychotropic drugs prescribed was reduced by 28\% (from 636 before to 458 after the intervention). The mean number of psychotropic drugs prescribed per patient decreased from 2.71 at baseline to 1.95 at 1 month postintervention and 2.01 at 6 months ( $p<0.001$ for both time points). Antipsychotics were the drug class showing the highest reduction rate (49.66\%). Reintroduction of discontinued psychotropic drugs was $2 \%$ at 1 month following the intervention and $12 \%$ at 6 months.
\end{abstract}

Conclusions: A consensus guidelines-based therapeutic intervention with a patient-centered medication review by a multidisciplinary team led to a reduction in prescription of psychotropic drugs in institutionalized dementia patients.

Keywords: Medication review, Dementia, Psychotropic drugs, Nursing homes, Institutionalized patients

\footnotetext{
* Correspondence: mmassot.mn.ics@gencat.cat

${ }^{1}$ Servei d'Atenció Primària Vallès Occidental, Institut Català de la Salut,

Rambla Sabadell 229, 08201, Sabadell, Barcelona, Spain

Full list of author information is available at the end of the article
}

(c) The Author(s). 2019 Open Access This article is distributed under the terms of the Creative Commons Attribution 4.0 International License (http://creativecommons.org/licenses/by/4.0/), which permits unrestricted use, distribution, and reproduction in any medium, provided you give appropriate credit to the original author(s) and the source, provide a link to the Creative Commons license, and indicate if changes were made. The Creative Commons Public Domain Dedication waiver (http://creativecommons.org/publicdomain/zero/1.0/) applies to the data made available in this article, unless otherwise stated. 


\section{Background}

Patients with dementia often show neuropsychiatric symptoms. These are known as behavioral and psychological symptoms of dementia (BPSD) and are a common motive for medical consultations, hospital admissions, and nursing home stays [1]. It is estimated that at least $90 \%$ of dementia patients will develop some type of BPSD over a period of 5 years, and this may have significant clinical implications in $85 \%$ of cases [2-4].

It is known that psychotic symptoms are more frequent during the moderate and severe stages of dementia, but the relationship between depression and dementia is not as clear. Some studies have suggested that there may be an inverse relationship, with depression present in the initial stages of the illness and absent in later ones. However, this notion may result from the difficulty in diagnosing depression in advanced stages of dementia, and not from an actual reduction in its prevalence [4]. Study of the natural history of dementia has shown that cognitive deterioration occurs more rapidly in patients with psychotic symptoms [5]. When these are treated with medication, periodic review of the treatment may be necessary [6], as the patient's clinical status may change quickly, and along with it, the associated BPSD.

Psychotropic drug use in patients with dementia is currently quite high $[7,8]$. An estimated $70 \%$ of dementia patients take 1 or more of these drugs [8-10]. In individuals over the age of 65 , the prevalence of benzodiazepine consumption is $20 \%$ [11] and prescription of antidepressant agents is $40 \%$ [3]. In institutionalized dementia patients older than 65 years, benzodiazepine prescription reaches $47.1 \%$ and antidepressants $54 \%$, whereas the use of antipsychotic drugs is estimated at 24.8 to $39.9 \%$ [8, 12].

Extensive use of psychotropic drugs to treat BPSD in institutionalized patients may lead to decreased cognitive capacity, rigidity, or somnolence, and result in complications such as pneumonia [13]. The EARCAS study [14], designed to explore the magnitude and implications of incidents related to patient safety in Spanish nursing homes, concluded that drug-related adverse events may be linked to a lack of medication review, with falls being the main adverse event. In addition, $96.6 \%$ of the experts consulted stated that the patients at the highest risk of experiencing a drug-related adverse event are those with impaired cognitive function (eg, Alzheimer disease, coma, dementia), and particularly those with behavioral disorders. In parallel, Billioti et al. [11] found that benzodiazepine use is associated with an increased risk (around 50\%) of experiencing dementia. In a cohort study including 60,746 patients, Coupland et al. [15] examined the association between antidepressant use and serious drug-related adverse events in patients older than 65 years with depression. The authors reported a significantly higher rate of all-cause mortality in the group treated with antidepressants than in those who did not receive these drugs.

Several studies $[16,17]$ have cautioned about the risks of psychotropic drug use for treating BPSD and the need to periodically review the therapeutic strategy applied over time. This concern suggests that there may be a need for interventions aimed at reducing the risk associated with these drugs. The recent publication of several clinical trial protocols [18-21] intended to decrease the use of these drugs in institutionalized dementia patients further illustrates the growing interest in this issue.

Considering this background, the aim of this study was to design an intervention by consensus between specialized caregivers implicated in the management of patients with dementia (eg, neurologists, geriatric specialists) and primary care general practitioners (GPs) for optimizing and potentially reducing the prescription of psychotropic drugs in this population, and evaluate the changes occurring after its implementation.

\section{Methods}

This is a prospective, multicenter, quasi-experimental, longitudinal, pre/postintervention study, conducted between 2012 and 2014. The patients included were residing in 7 nursing homes linked to a single primary care center in a semi-urban setting; the total population was 606 institutionalized patients, 40\% with dementia. Drug prescription from the publically-funded national health system was carried out by the GPs in the primary care center. Dementia patients in these homes receiving pharmacological treatment with 1 or more psychotropic drugs from the Anatomical Therapeutic Chemical Classification System of the WHO [22] (ATC code N, with the exception of N01 anesthetics, N02 analgesics, and N07 others) for 3 months or longer were included. Patients with an active diagnosis of severe mental disorder (eg, personality disorder, severe depression, psychosis, schizophrenia) were excluded from the study. At 6 months following the intervention, all patients were assessed to determine whether some of the drugs that were discontinued during the intervention had been restarted or new drugs prescribed.

The main study variable was the number of psychotropic drugs prescribed for each patient before the intervention, immediately after the intervention, and at 1 month and 6 months of follow up. Secondary variables included the patients' socio-demographic characteristics (age and sex) and drug-related variables (active ingredient, dose, and frequency of administration of all psychotropic drugs prescribed).

The intervention consisted of a review of the medications received by the participating patients, accomplished by individual, personal meetings between 1 primary care GP and 1 pharmacist, and the nursing 
home physicians and nurses. The intervention was developed as follows:

1) Creation of BPSD guidelines by consensus (Additional file 1). A multidisciplinary group of referral professionals was formed, consisting of a neurologist, a psychiatrist, a geriatrician, 2 GPs and 4 pharmacists, who designed the therapeutic guidelines for treating BPSD. The guidelines were evidence-based and included individual recommendations for pharmacological and nonpharmacological management of the behavioral and psychological symptoms, grouped as follows: apathy, aggression, anxiety, agitation, depression, psychosis, and insomnia. A brief summary of the general content of the guidelines and the method used in the medication review is provided in Additional file 2.

2) Prior to implementation of the guidelines-based medication review, $1 \mathrm{GP}$ and 1 primary care pharmacist underwent a training phase focused on management of patients with BPSD (eg, assessment of dementia patients, management of the medication implicated in their treatment, the criteria and scheduling for drug withdrawal, strategies for symptoms that did not respond to treatment). Both these professionals had participated in designing the guidelines and were in charge of carrying out the fieldwork in the nursing homes. The training was carried out in person, was led by a neurologist, geriatrician, and psychogeriatrician, and was based on the contents of the guidelines. In addition to discussion about the theoretical framework, several case examples were raised and examined. A practical training session was then carried out in institutionalized dementia patients prescribed psychotropic drugs, where the trainees applied what had been discussed during the training before the start of the project.

3) Review of the treatment plan: A timeline for nursing home visits was created to review the course of the treatment. Before each scheduled visit (work session) with the nursing home physician and nurse, the medication patients were receiving underwent a preliminary evaluation by the GP and pharmacist to detect any incidents related to the prescription (eg, duplicates, inappropriate drugs) and to determine which prescription-related aspects required evaluation of patients for decision-making (continue or discontinue a drug).

4) Work session in the nursing home: Before conducting the joint medication review, the primary care GP and pharmacist asked the nursing home physician and nurse to confirm the patients' dementia diagnosis to include them in the study. These professionals then established the patients' current status with regard to their prognosis, level of dependence, and frailty to facilitate decisions on the need to adapt the intensity of treatment, change treatment, or discontinue treatment based on the benefit-risk for the patient [23]. The assessment of the patient's current status included the severity of dementia (Global Dementia Scale [GDS]), level of dependence (Barthel index score), cognitive state (Pfeiffer test), and prognosis (in end-of-life patients). Treatment assessment included the indication, effectiveness, and safety (eg, duplication, interactions, contraindications) of the drugs prescribed. Based on these joint evaluations, changes in the patient's treatment plan considered to be appropriate and relevant were proposed.

5) Follow-up: Follow-up of the consensus changes was carried out at 1 and 6 months to quantify the reintroduction of psychotropic drugs that had been discontinued during the work sessions, changes in the dose of psychotropic drugs that continued in prescription, and prescription of any new psychotropic drug.

Patient-related data were provided by the nursing home professionals and prescription data were obtained from the patients' medical records. The information was entered in an anonymous database by one of the authors (MTC) at each of the study time points. The data collected were reviewed to ensure accurate recording.

\section{Statistical analysis}

In the univariate analysis, quantitative variables are expressed as the mean and standard deviation (SD) and qualitative variables as the percentage. The Student-t test for paired data was used to compare the mean number of psychotropic drugs prescribed before (baseline) and after the intervention and between baseline and 1 and 6 months. The Wilcoxon test was used to analyze this variable in each nursing home. The sample size was not calculated a priori, as all patients meeting the inclusion criteria were enrolled. The G*power program [24] was used to analyze the statistical power of the study a posteriori, to determine whether there was a loss of power due to the sample size.

\section{Results}

From a total population of 606 institutionalized individuals, 250 patients had a diagnosis of dementia; 240 of the 250 (96\%) were receiving 1 or more psychotropic drugs for at least 3 months and were included in the study Over follow-up, 2 patients (1.67\%) were lost at 1 month and 22 patients (10\%) were lost at 6 months due to death or a change of nursing home (Fig. 1).

Mean patient age was 87.09 years (SD: 6.795), and 75\% $(n=180)$ were women. There were no significant differences 
606 institutionalized patients from 7 nursing

homes (dementia prevalence, $40 \%$ )

Review of the therapeutic plans by the GP and

$\mathrm{PCP}$ before the NH session to detect

prescription-related incidents and to know

what aspects related to the prescription were

required in the joint assessment of individual

patients

Sequential timeframe of interviews with the $\mathrm{NH}$ physician and nurse

Patient assessment provided by NH professionals: Diagnosis, Barthel, Pfeiffer, GDS and prognosis. Confirmation of the dementia diagnosis for inclusion in the study.

Patients without dementia or with SMD

Dementia patients without psychiatric drug prescription (10)

$\mathbf{N}=\mathbf{2 4 0}$ met the inclusion criteria

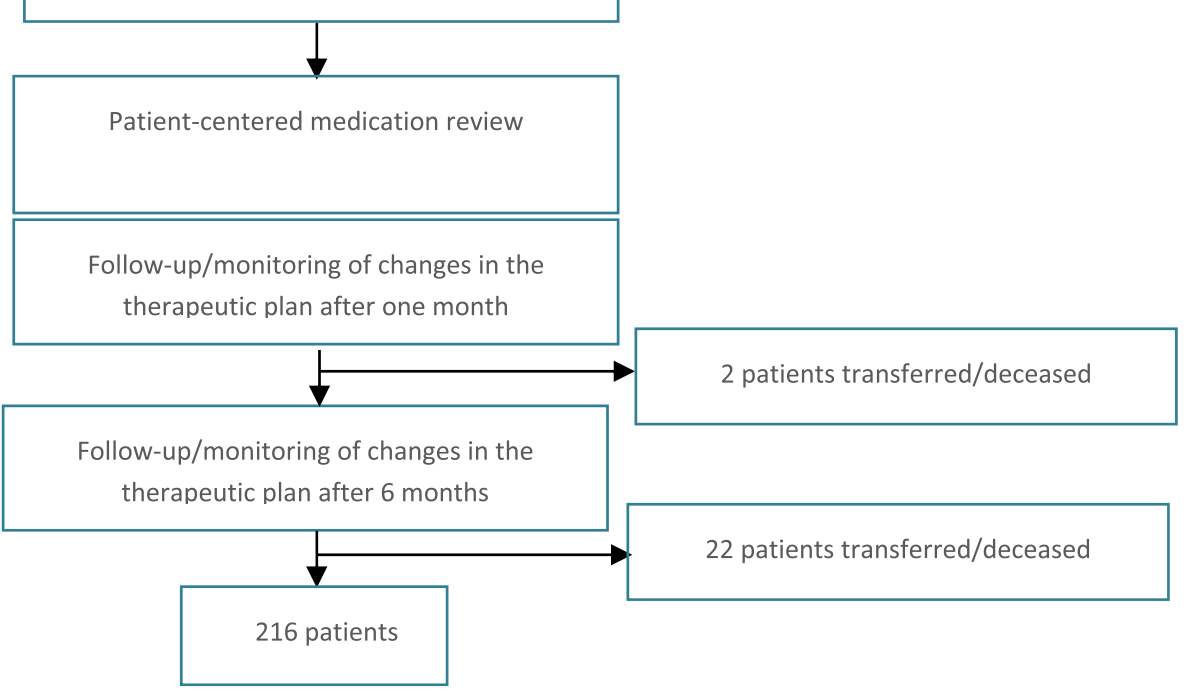

Fig. 1 Flow chart of patient inclusion at each point of the intervention. Abbreviations: GDS Global Dementia Scale, GP general practitioner, NH nursing home, PCP primary care pharmacist, SMD severe mental disorder

in patient age between the participating nursing homes. The characteristics of the study population in each nursing home are shown in Table 1.
In total, 636 psychotropic drugs were prescribed before the intervention and 458 after; this latter value includes 216 drugs that were deprescribed and 38 new 
Table 1 Patient age and sex, and percentage of patients with dementia in each nursing home

\begin{tabular}{lllll}
\hline Nursing home & $\begin{array}{l}\text { Patients with dementia } \\
\text { and PD, } n(\%)\end{array}$ & $\begin{array}{l}\text { Prevalence of } \\
\text { dementia, } \%\end{array}$ & Mean age, y (SD) & Women, $n(\%)$ \\
\hline NH 1 & $35(14.6 \%)$ & $35.0 \%$ & $87.98(7.78)$ & $28(80 \%)$ \\
NH 2 & $42(17.5 \%)$ & $43.8 \%$ & $88.05(4.99)$ & $38(90.5 \%)$ \\
NH 3 & $25(10.4 \%)$ & $24.0 \%$ & $87.44(6.25)$ & $15(60 \%)$ \\
NH 4 & $14(5.8 \%)$ & $35.0 \%$ & $89.57(8.22)$ & $10(71.4 \%)$ \\
NH 5 & $52(21.7 \%)$ & $45.2 \%$ & $85.25(7.51)$ & $32(61.5 \%)$ \\
NH 6 & $50(20.8 \%)$ & $58.1 \%$ & $86.66(7.35)$ & $37(74 \%)$ \\
NH 7 & $22(9.2 \%)$ & $33.9 \%$ & $87.36(3.63)$ & $20(90.9 \%)$ \\
TOTAL & 240 & $39.6 \%$ & $87.9(6.8)$ & $180(75 \%)$ \\
\hline
\end{tabular}

NH nursing home, PD psychotropic drugs, SD standard deviation, y years. Patients with dementia: patients evaluated by a neurologist with a confirmed diagnosis of dementia according to ICD-10 criteria

drugs that were prescribed; therefore, the final balance was 178 psychotropic drugs discontinued $(28 \%$ decrease). The decrease was statistically significant $(p<$ $0.0001)$ at all the time points analyzed relative to baseline. The pre- and postintervention difference in the number of drugs prescribed per patient was $0.755(95 \%$ CI: 0.624-0.886); that is, 0.771 (95\% CI: $0.635-0.908)$ at 1 month of follow-up and 0.634 (95\% CI, 0.474-0.794) at 6 months ( $p=0.000$ in all cases). The medication changes occurring in relation to the intervention, including dosing changes, are summarized in Table 2. The decrease in number of psychotropic drugs per patient was statistically significant in all the participating nursing homes except one (Table 3). At 1 month after discontinuation, only 4 (2\%) psychotropic drugs that had been discontinued were reintroduced, and at 6 months, $12 \%$ of drugs were restarted (11 antidepressants, 8 antipsychotics, and 3 hypnotics).
In the analysis according to drug class, antipsychotics were the class most frequently discontinued (49.66\%), followed by acetylcholinesterase inhibitors and memantine (28.57\%), and antidepressants (28.48\%). However, hypnotics such as clomethiazole, hydroxyzine, and zolpidem were only reduced by $15.85 \%$. The changes in psychotropic drug prescription by drug class along the study are shown in Fig. 2.

\section{Discussion}

Patient-centered medication review, as conducted in this study with the support of dedicated therapy guidelines, achieved an overall 28\% reduction in psychotropic drug prescription, which remained at an acceptable level at 6 months of follow-up for all the drug classes examined. The reductions were particularly marked for anticholinesterase inhibitors and antidepressant agents.

Table 2 Overall prescription-related changes following the intervention, and at 1 and 6 months

\begin{tabular}{|c|c|c|c|c|}
\hline Drug prescription changes & Baseline & Postintervention & 1 month & 6 months \\
\hline Dose increase, $n$ & & 25 & 2 & 9 \\
\hline Dose decrease, $n$ & & 34 & 11 & 6 \\
\hline Discontinued, $\mathrm{n}$ & & 216 & 15 & 43 \\
\hline Newly prescribed drugs, $n$ & & 38 & 9 & 42 \\
\hline Reintroduced drugs, n & & 0 & 4 & 22 \\
\hline Patients 0 PD, n (\%) & $10(4.00 \%)$ & $32(12.80 \%)$ & $33(13.31 \%)$ & $25(11.06 \%)$ \\
\hline Patients 1-2 PD, n (\%) & $122(48.80 \%)$ & $151(60.40 \%)$ & $144(58.06 \%)$ & 134 (59.29\%) \\
\hline Patients 3-4 PD, n (\%) & $89(35.60 \%)$ & $60(24.00 \%)$ & $65(26.21 \%)$ & $55(24.34 \%)$ \\
\hline Patients > 4 PD, n (\%) & $29(11.60 \%)$ & 7 (2.80\%) & $6(2.42 \%)$ & $12(5.31 \%)$ \\
\hline $\begin{array}{l}\text { Number psychotropic } \\
\text { drugs/patient, mean (SD) }\end{array}$ & $2.71(1.47)$ & $1.95(1.24)$ & $1.95(1.26)$ & $2.06(1.36)$ \\
\hline Difference (95\% Cl) & \multicolumn{2}{|c|}{$0.755(0.624-0.886)^{*} p=0.000$} & $\begin{array}{l}0.771(0.635-0.908)^{*} \\
p=0.000\end{array}$ & $\begin{array}{l}0.634(0.474-0.794)^{*} \\
p=0.000\end{array}$ \\
\hline
\end{tabular}

Prescription-related changes and percentages of patients receiving psychotropic agents grouped into 4 categories $(0,1-2,3-4$, and $>4$ psychotropic drugs) at the different time points. The calculation of percentages in each of the 4 categories was made over the total of patients with dementia in the nursing homes $(n=250)$. Difference in the mean number of psychotropic drugs prescribed before (baseline) and after the intervention and between baseline and 1 and 6 months are shown.

*Difference in the mean number of psychotropic drugs prescribed before (baseline) and after the intervention and between baseline and 1 and 6 months 
Table 3 Number of psychotropic drugs per patient at the different assessment points in each nursing home

\begin{tabular}{|c|c|c|c|c|}
\hline \multirow[b]{2}{*}{ Nursing home $(\mathrm{NH})$} & \multicolumn{4}{|c|}{ Number psychotropic drugs/patient } \\
\hline & Baseline, mean (SD) & Post-intervention, mean (SD) & 1 month, mean (SD) & 6 months, mean (SD) \\
\hline \multirow[t]{2}{*}{$\mathrm{NH} 1$} & $2.71(1.53)$ & $2.06(1.33)$ & $2.06(1.33)$ & $2.32(1.66)$ \\
\hline & $0.65(p=0.001)$ & & $0.65(p=0.001)^{*}$ & $0.39(p=0.035)^{*}$ \\
\hline \multirow[t]{2}{*}{$\mathrm{NH} 2$} & $2.64(1.30)$ & $1.83(1.14)$ & $1.93(1.21)$ & $2.12(1.35)$ \\
\hline & $0.81(p=0.000)$ & & $0.71(p=0.000)^{*}$ & $0.52(p=0.002)^{*}$ \\
\hline \multirow[t]{2}{*}{$\mathrm{NH} 3$} & $3(1.53)$ & $2.24(1.42)$ & $2.24(1.36)$ & $1.95(1.40)$ \\
\hline & $0.76(p=0.000)$ & & $0.76(p=0.001)^{*}$ & $1.05(p=0.000)^{*}$ \\
\hline \multirow[t]{2}{*}{$\mathrm{NH} 4$} & $3.43(1.87)$ & $2.43(1.65)$ & $2.14(1.70)$ & $2.54(1.94)$ \\
\hline & $1.00(p=0.004)$ & & $1.29(p=0.004)^{*}$ & $0.89(p=0.008)^{*}$ \\
\hline \multirow[t]{2}{*}{ NH 5} & $3.1(1.49)$ & $1.94(1.13)$ & $1.98(1.15)$ & $2.04(1.17)$ \\
\hline & $1.16(p=0.000)$ & & $1.12(p=0.000)^{*}$ & $1.06(p=0.000)^{*}$ \\
\hline \multirow[t]{2}{*}{$\mathrm{NH} 6$} & $2.23(1.26)$ & $1.62(1.04)$ & $1.55(1.14)$ & $1.64(0.98)$ \\
\hline & $0.61(p=0.000)$ & & $0.68(p=0.000)^{*}$ & $0.59(p=0.000)^{*}$ \\
\hline \multirow[t]{2}{*}{$\mathrm{NH} 7$} & $2.23(1.45)$ & $2.14(1.32)$ & $2.14(1.32)$ & $2.23(1.54)$ \\
\hline & $0.09(p=0.593)$ & & $0.09(p=0.593)^{*}$ & $0.00(p=1.000)^{*}$ \\
\hline
\end{tabular}

*Difference in the mean number of psychotropic drugs prescribed before (baseline) and after the intervention and between baseline and 1 and 6 months

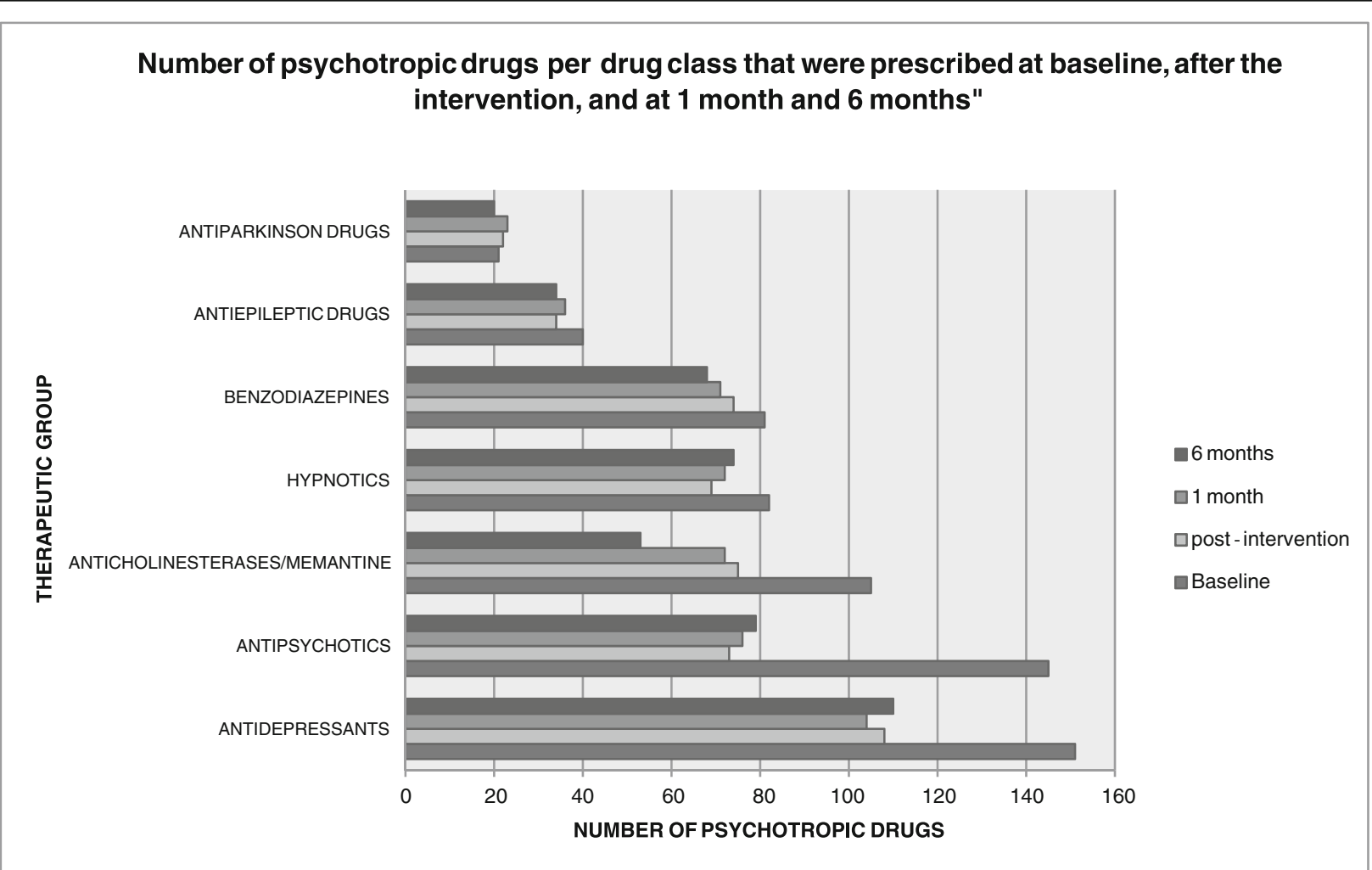

Fig. 2 Number of psychotropic drugs per drug class that were deprescribed al baseline after the intervention, and at 1 month and 6 months 
The decrease in prescribed psychotropic drugs achieved in this study was greater than that reported by other authors. In a clinical trial [25], Fossey et al. described a $19.1 \%$ reduction in nursing home residents with severe dementia. The differing values may be due to differences in the study designs and populations included. In the report by Fossey et al., deprescription was only contemplated for neuroleptic agents. Although neuroleptics were the drug class most commonly deprescribed in the present study, deprescription of any psychotropic drug was included, based on the patient's response to treatment and current clinical status with regard to comorbidity, degree of dependence, and functionality. In a study by van der Spek [26], biannual medication review was applied to improve the appropriateness of the psychotropic drugs prescribed in nursing home patients with dementia. As the evaluation of the intervention differed from the one used here, the significant results obtained in improving the appropriateness of psychotropic drugs prescription cannot be compared with those of our study. However, the two studies coincide in the conclusion that a structured medication review by a multidisciplinary team can help to optimize psychotropic drug prescription in these patients. Other studies in nursing home patients (although not specifically dementia patients) $[27,28]$ have also provided evidence that multidisciplinary medication review can reduce the prescription of psychotropic drugs in institutionalized patients.

The fact that acetylcholinesterase inhibitors and memantine were the second most frequently deprescribed agents suggests a considerable percentage of patients with severe dementia-that is, showing a GDS stage of $7 \mathrm{~b}$ or greater [29]. The study by Hogan and Strafford in patients with Alzheimer's disease [30] emphasized the need for individualized assessment in the decision to deprescribe these drugs, even though there is consensus for their withdrawal in advanced stages of dementia.

At the 1-month follow-up, only 4 patients were restarted on previously discontinued psychotropic drugs, which could indicate that the patient-centered multidisciplinary approach used here was effective for optimizing and reducing prescription of psychotropic drugs. At 6 months, drugs were restarted in 22 patients, and in the case of anti-depressants, this may have been done in response to worsening of the depression symptoms. Although this clinical aspect was not specifically assessed in our study, it would coincide with the conclusion of Bergh et al. [31] that withdrawal of antidepressants in patients with dementia could worsen their depression symptoms, and that re-introduction of these drugs would be advisable. However, reintroduction of antipsychotic agents at 6 months could be due to the reappearance of symptoms once the patient had stabilized, as was suggested in the study by Devanand et al. [32]. This study concluded that discontinuation of antipsychotic agents can increase the probability that these symptoms will reappear. However, it is also important to note that reducing the exposure time to antipsychotic drugs can lower the probability of an adverse drug-related reaction [6]. The increase in the mean number of psychotropic drugs prescribed at 6 months following the intervention could have been due not only to reintroduction of some previous treatments, but also to the prescription of new treatments in order to control the appearance of new symptoms related to the evolution of the illness.

Following the intervention, a statistically significant reduction in prescribed psychotropic drugs occurred in all nursing home except one, in which the decrease was smaller and non-significant. The mean number of drugs used per patient in this center did not differ from that of the other nursing homes, but $82 \%$ of the patients were receiving 3 or fewer drugs, and our study results showed that prescription changes were more prevalent in patients receiving 4 or more drugs. In addition, $39 \%$ of the psychotropic drugs used were antiepileptic agents or specific medication for treating dementia. Along the intervention, antiepileptics were the drug class showing the smallest reduction, and dementia-specific treatment was generally considered appropriate and not a candidate for discontinuation. These factors may have played a part in the smaller prescription decrease observed.

Regarding the limitations of the study, we should note that it is a pre/post study with no control group. In selecting this design, we took into consideration the growing evidence of psychotropic drug-related adverse effects in patients with dementia [14-17] [29, 30]. The design was considered to be a pragmatic primary health care approach. When the intervention was being considered, we saw that the prevalence of psychotropic drug use in our dementia patients was higher than that described by Gustafsson [8] and other authors [9, 10]. We believed that the intervention would improve the routine management of this population, and a control group was not contemplated so that all individuals participating could benefit from it. As all dementia patients eligible for the intervention were included, we did not calculate the sample size a priori. However, at completion of the study, a power analysis was performed using the G*power program [24], which yielded a statistical power greater than $80 \%$.

Another limitation was that no further clinical evaluations were carried out after the initial patient assessment; only medication-related changes were recorded. However, we were able to show that deprescription decisions remained in the sample over some months. Furthermore, selection of the nursing homes to be included in the study was not randomized. The intervention was proposed in all large nursing homes in the catchment 
area of the primary care team, and some chose not to participate. All the candidate nursing homes were of a general nature (ie, they were not centers specialized in certain conditions) and there were no significant differences in the age of the residents between those that participated or not in the study. However, the participating centers cannot be considered representative of all nursing homes in our setting. Finally, the medication review took into account the patient's health status, frailty, and prognosis, but the patient did not take part in the treatment decisions.

\section{Conclusion}

In conclusion, an intervention based on dedicated, evidence-based therapeutic guidelines designed by a multidisciplinary team and implemented by consensus, together with a patient-centered clinical medication review, led to a reduction in prescription of psychotropic drugs in institutionalized dementia patients. As this illness and its associated symptoms may vary considerably over time, it is important to review the patient's medication on an individual basis periodically to ensure that it is appropriate according to benefit/risk criteria.

\section{Additional files}

Additional file 1: Therapeutic guideline for treating BPSD used in this study (written in Catalan). (PDF $306 \mathrm{~kb}$ )

Additional file 2: Brief summary of the consensus guidelines and development of the pharmacological review intervention for the management of BPSD. (PDF $46 \mathrm{~kb}$ )

\section{Abbreviations}

ATC: The Anatomical Therapeutic Chemical Classification System of the World Health Organization; BPSD: Behavioral and psychological symptoms of dementia; GDS: Global Dementia Scale; GP: General practitioner; ICS: Institut Català de la Salut (Catalan Health Institute); IDIAPJGol: Fundació Institut Universitari per a la recerca a l'Atenció Primària de salut Gol i Gurina (Jordi Gol Primary Care Research Institute); NH: Nursing home; PD: Psychotropic drugs; SD: Standard deviation; SMD: Severe mental disorder

\section{Acknowledgements}

This study was awarded best poster communication in the European General Practice Research Network (EGPRN) [33] and the Spanish Society of Primary Care Pharmacists (SEFAP) congresses. It also received the VII Chiesi Award for Primary Care Pharmaceutical Research. The authors thank Celine Cavallo for English language support.

\section{Funding}

No funding was received.

\section{Availability of data and materials}

The datasets generated and /or analyzed during the current study are not publicly available due politics of our institution but are available from the corresponding author on reasonable request. We added the therapeutic guideline for treating BPSD used in this study (written in Catalan) and a summary table of contents as supplementary data.

\section{Authors' contributions}

MM, PT and MT carried out the study design and supervised the data collection. MM, MT, IG, AF, OH and PT contributed to the data analysis, writing of the manuscript, data interpretation, discussion and answers to the Reviewers. All authors have read and approved the final manuscript.

\section{Competing interest}

The author declares that they have no competing interests.

\section{Ethics approval and consent to participate}

The study protocol was approved by the Ethics and Clinical Research Committee of the Jordi Gol Primary Care Research Institute (IDIAPJGol, Fundació Institut Universitari per a la recerca a I'Atenció Primària de salut Gol i Gurina) of the Catalan Health Institute (ICS, Institut Català de la Salut, Code: P15/120). The IDIAPJGol supervises all primary care research activity within the ICS. General Practitioners who participated in the implementation of the intervention were members of the research team and verbally consent their participation. Informed consent for participation was not required from the patients, as the intervention was focused on the prescribing doctors, not on the patients, themselves

\section{Consent for publication}

Not applicable.

\section{Publisher's Note}

Springer Nature remains neutral with regard to jurisdictional claims in published maps and institutional affiliations.

\section{Author details}

${ }^{1}$ Servei d'Atenció Primària Vallès Occidental, Institut Català de la Salut, Rambla Sabadell 229, 08201, Sabadell, Barcelona, Spain. Equip d'Atención Primària Arenys de Mar, Institut Català de la Salut, Arenys de Mar, Barcelona, Spain. ${ }^{3}$ Unitat de Suport a la Recerca Metropolitana Nord, Fundació Institut Universitari per a la recerca a l'Atenció Primària de Salut Jordi Gol i Gurina (IDIAPJGol), Mataró, Spain.

Received: 12 February 2018 Accepted: 13 December 2018

Published online: 08 January 2019

\section{References}

1. Scottish Intercollegiate Guidelines Network. Management of patients with dementia. In: A national clinical guideline; 2006.

2. Steinberg $M$, et al. Point and 5-year period prevalence of neuropsychiatric symptoms in dementia: the Cache County study. Int J Geriatr Psychiatry. 2008;23:170-7.

3. Bergh S, Selbaek G, Engedal K. Discontinuation of antidepressants in people with dementia and neuropsychiatric symptoms (DESEP study): double blind, randomized, parallel group, placebo controlled trial. BMJ. 2012;344:e1566.

4. García-Alberca JM, Lara Muñoz P, Berthier Torres M. Neuropsychiatric and behavioral symptomatology in Alzheimer disease. Actas Esp Psiquiatr. 2010; 38(4):212-22.

5. McShane R, Gedling K, Keene J, et al. Getting lost in dementia: a longitudinal study of a behavioral symptom. Int Psychogeriatr. 1998;10(3): 253-60.

6. Gómez-Pavón J, González García P, Francés Román I, et al. Recomendaciones en la prevención de reacciones adversas a medicamentos en personas mayores con demencia. Rev Esp Geriatr Gerontol. 2010;45(2):89-96

7. Gustafsson M, Karlsson S, Lövheim H. Inappropriate long-term use of antipsychotic drugs is common among people with dementia living in specialized care units. BMC Pharcology and Toxicology. 2013;14:10.

8. Gustafsson M, Karlsson S, Lövheim H. Psychotropic drug use among people with dementia - a six-month follow-up study. BMC Pharcology and Toxicology. 2013;14:56

9. Azermai M, Elseviers M, Petrovic M, Van Bortel L, Vander Stichele R. Geriatric drug utilization of psychotropics in Belgian nursing homes. Hum Psychopharmacol Clin Exp. 2011;26:12-20.

10. Gobert M, D'hoore W. Prevalence of psychotropic drug use un nursing homes for the aged in Quebec and in French-speaking area of Switzerland. Int J Geriatr Psychiatry. 2005;20:712-21.

11. Billioti de Gage S, Bégaud B, Bazin F, Verdoux H, Dartigues JF, Pérès K, Kurth T, Pariente A. Benzodiazepine use and risk of dementia: prospective population-based study. BMJ. 2012;345:e6231. 
12. Banerjee $\mathrm{S}$. The use of antipsychotic medication for people with dementia: time for action. A report for the minister of state for care services. London: Department of Health; 2009.

13. Tampi RR, Tampi DJ, Balachandran S, Srinivasan S. Antipsychotic use in dementia: a systematic review of benefits and risks from meta-analyses. Ther Adv Chronic Dis. 2016;7(5):229-45.

14. Estudio EARCAS. Eventos Adversos en Residencias geriátricas y centros Asistenciales Sociosanitarios. In: Madrid: Ministerio de Sanidad, Política Social e lgualdad; 2011

15. Coupland C, Dhiman P, Morriss R, Arthur A, Barton G, Hippisley-Cox J. Antidepressant use and risk of adverse outcomes in older people: population based cohort study. BMJ. 2011;343:d4551.

16. Ballard C, Hanney ML, Theodoulou M, Douglas S, McShane R, Kossakowski K, Gill R, Edmund Juszczak E, Yu LM, Jacoby R. For the DART-AD investigators. The dementia antipsychotic withdrawal trial (DART-AD): long-term follow-up of a randomised placebo-controlled trial. Lancet Neurol. 2009;8:151-7.

17. Corbett A, Burns A, Ballard C. Don't use antipsychotics routinely to treat agitation and aggression in people with dementia. BMJ. 2014;349:96420. https://doi.org/10.1136/bmj.g6420.

18. Pitkala $\mathrm{KH}$, Juola $\mathrm{AL}$, Soini $\mathrm{H}$, Laakkonen ML, Kautiainen $\mathrm{T}-\mathrm{G}$, Finne-Soveri $H$, Bjorkman M. Reducing inappropriate, anticholinergic and psychotropic drugs among older residents in assisted living facilities: study protocol for a randomized controlled trial. Trials. 2012;13:85.

19. Whitaker R, Ballard C, Stanfford J, Orrell M, Moniz-Cook E, Woods RT, Murray J, Knapp M, Woodward Carlton B, Fossey J. Feasibility study of an optimized person-centered intervention to improve mental health and reduce antipsychotics amongst people with dementia in care homes: study protocol for a randomized controlled trial. Trials. 2013;14:13.

20. Richter C, berg A, Fleischer S, Köpke S, Balzer K, Fick EM, Sönnichsen A, Löscher S, Vollmar HC, Haastert B, Icks A, Dintsios CM, Mann E, Wolf U, Meyer $G$. Effect of person-centered care on antipsychotic drug use in nursing homes (EPCentCare): study protocol for a cluster-randomised controlled trial. Implement Sci. 2015;10:82

21. Smeets C, Smalbrugge M, Gerritsen DL, Nelissen-Vrancken M, Wetzels RB, van der Speck K, Zuidema SU, Koopmans R. Improving psychotropic drug prescription in nursing home patients with dementia: desing of a cluster randomized controlled trial. BMC Psychiatry. 2013;13:280.

22. WHO Collaborating Centre for Drug Statistics Methodology. ATC index. https://www.whocc.no/atc_ddd_index/. Accesed 31 July 2018.

23. American Geriatrics Society Expert Panel on the Care of Older Adults with Multimorbidity. Patien-centered Care for older adults with Multiple Chronic Conditions: A Stepwise Approach from the American geriatrics Society. J Am Geriatr Soc 2012. Special article. 1-12.

24. Faul F, Erdfelder E, Lang AG, Buchner A. G*power 3: a flexible statistical power analysis program for the social, behavioral, and biomedical sciences. Behavior Research Methds. 2007;39(2):175-91.

25. Fossey J, Ballard C, Juszczak E, James I, Alder N, Jacoby R, Howard R. Effect of enhanced psychological care on antipsychotic use in nursing home residents with severe dementia: cluster randomized trial. BMJ. 2006. https:// doi.org/10.1136/bmj.38782.575868.7C.

26. Van der Spek K, Koopmans R, Smalbrugge M, Nelissen-Vrancken M, Wetzels R, Smeets C, Vries E, Teerenstra S, Zuidema SU, Geritsen DL. The effect of biannual medication reviews on the appropriateness of psychotropic drug use for neuropsychiatric symptoms in patients with dementia: a randomized controlled trial. Age Ageing. 2018;47:430-7.

27. Patterson SM, Hughes C, Crealey G, Cardwell C, Lapane KL. An evaluation of an adapted US model of pharmaceutical care to improve psychoactive prescribing for nursing home residents in Northern Ireland (Fleetwood Northern Ireland study). J Am Geriatr Soc. 2010;58:44-53.

28. Westbury JL, Gee P, Ling T, Brown DT, Franks KH, Bindoff I, Bindoff A, Peterson GM. RedUSe; reducing antipsychotic and benzodiazepine prescribing in residential aged care facilities. Med J Aust. 2018;208(9): 398-403.

29. Buckley JS, Salpeter SR. A risk-benefit assessment of dementia medications: systematic review of the evidence. Drugs Aging. 2015;32:453-67.

30. Hogan DB, Strafford B. Long-term efficacy and toxicity of cholinesterase inhibitors in the treatment of Alzheimer disease. CanJPsychiatry. 2014;59(12):618-23.

31. Bergh S, Selbæk G, Engedal K. Discontinuation of antidepressants in people with dementia and neuropsychiatric symptoms (DESEP study): double blind, randomised, parallel group, placebo controlled trial. BMJ. 2012:344:e1566. https://doi.org/10.1136/bmj.e1566.
32. Devanand DP, Mintzer J, Schultz SK, Andrews HF, Sultzer DL, de la Pena D, Gupta S, Colon S, Schimming C, Pelton GH, Levin B. Relapse risk after discontinuation of risperidone in Alzheimer's disease. N Engl J Med. 2012; 367:1497-507.

33. Massot M, Tristany M, Franzi A, Garcia I. Reducing the number of antidepressants, antipsychotics and benzodiazepines in nursing home residents with dementia. European Journal of General Practice. 2016;2(22):147-54.
Ready to submit your research? Choose BMC and benefit from:

- fast, convenient online submission

- thorough peer review by experienced researchers in your field

- rapid publication on acceptance

- support for research data, including large and complex data types

- gold Open Access which fosters wider collaboration and increased citations

- maximum visibility for your research: over $100 \mathrm{M}$ website views per year

At BMC, research is always in progress.

Learn more biomedcentral.com/submissions 